\title{
Invarianza Factorial del Cuestionario sobre Creencias de Autoeficacia EACA en Universitarios de Ingeniería $Y$ Ciencias Sociales
}

\author{
Alejandro Chávez ${ }^{(1)}$, Martha Ornelas ${ }^{(1)}$, Belén De Rueda ${ }^{(2)}$ y Jesús Jasso ${ }^{(1)}$ \\ (1) Universidad Autónoma de Chihuahua. Facultad de Educación Física y Ciencias del Deporte, \\ DES Salud. Calle Escorza 900, CP 31000 Chihuahua, Chih.-México. \\ (2) Universidad Granada. Facultad de Ciencias de la Actividad Física y del Deporte, \\ Carretera de Alfacar, s/n CP: 18071 Granada-España. \\ (e-mail:achavezg@uach.mx, mornelas@uach.mx, belenvr@telefonica.nety jjasso@uach.mx)
}

Recibido Dic. 19, 2011; Aceptado Feb. 08, 2012; Versión final recibida Feb. 13, 2012

\begin{abstract}
Resumen
El presente estudio analiza las propiedades sicométricas de la Escala Autoeficacia en Conductas Académicas (EACA) en alumnos de Ingeniería y Ciencias Sociales. La muestra total fue de 1181 participantes; 592 de Ingeniería y 589 de Ciencias Sociales, con una edad media de 18.16 años y 18.24 años respectivamente. Los análisis factorial exploratorio y confirmatorio mostraron que una estructura trifactorial (Atención, Comunicación y Excelencia) es viable y adecuada para ambas poblaciones de acuerdo a los requisitos sicométricos establecidos cuando las respuestas al cuestionario son hechas por los propios alumnos. Además, la estructura factorial, las cargas factoriales y los interceptos se consideran invariantes en las dos poblaciones. Sin embargo, existen diferencias entre las poblaciones para las medias de los factores Comunicación y Excelencia.
\end{abstract}

\section{Factorial Invariance of the self-Efficacy Questionnaire EACA in University Students of Engineering and Social Sciences}

\begin{abstract}
The present study analyses the psychometric properties of the Self-Efficacy Scale in Academic Behavior (EACA) of students of Engineering and Social Sciences. The total sample comprised 1181 participants; 592 students of Engineering and 589 students of Social Sciences, with a mean age of 18.16 years and 18.24 years, respectively. Psychometric analysis showed that a threefactorial structure (Attention, Communication and Excellence) is feasible and adequate for both populations according to the established psychometric requirements when answers to the questionnaire are done by the students themselves. The results showed that factor structure, factor loadings and intercepts of the instrument could be considered invariant across groups. However, there are differences between groups for the averages of the factors Communication and Excellence.
\end{abstract}




\section{INTRODUCCIÓN}

Dentro de los contextos educativos ha existido un interés permanente por comprender los factores cognitivos y comportamentales que favorecen o dificultan el desempeño del estudiante en sus labores académicas y cómo el desempeño se relaciona con su desarrollo integral. En el área de la psicología educativa, específicamente, el constructo de autoeficacia ha recibido especial atención y se han generado importantes avances de investigación que han contribuido al mejoramiento de prácticas pedagógicas y de enseñanza (Pérez et al., 2011). La investigación empírica ha demostrado, de manera amplia, que la autoeficacia resulta ser más predictiva del rendimiento académico que otras variables cognitivas (Bandura, 1982), también que logra predecir el éxito posterior (Bandura, 1997; Ornelas et al., en prensa) y que es un importante mediador cognitivo de competencia y rendimiento (Vera et al., 2011) en cuanto favorece los procesos cognoscitivos (Carbonero y Merino, 2008; Ornelas et al., en prensa).

La propia motivación impulsa al ser humano a emprender conductas específicas en función de los logros que éste pretende alcanzar. Más no basta conocer con claridad aquello que deseamos lograr, ni tampoco el mejor medio para poder conseguirlo. Tampoco, basta con ser capaz de; es preciso juzgarse capaz de utilizar las capacidades y las habilidades personales ante circunstancias muy diversas. La percepción de las personas acerca de su propia eficacia se alza como un requisito fundamental para desarrollar con éxito las acciones conducentes al logro de los objetivos personales. Dicha autopercepción, denominada autoeficacia, ejerce una profunda influencia en la elección de tareas y actividades, en el esfuerzo y en la perseverancia de las personas cuando se enfrentan a determinados retos e incluso en las reacciones emocionales que experimentan ante situaciones difíciles (León-Rubio et al., 2011; Pérez et al., 2011; Wolters, 2004). En definitiva, las creencias de autoeficacia representan un mecanismo cognitivo que media entre el conocimiento y la acción y que determina, junto con otras variables, el éxito de las propias acciones (Castañeda et al., 2010; Pérez et al., 2011; Sansinenea et al., 2008; Zimmerman y Kitsantas, 2005).

En este trabajo se analiza la consistencia interna y la estructura factorial de un instrumento de autoreporte que permite identificar conductas académicas, cuyo nivel de autoeficacia percibido por los alumnos de nuevo ingreso representen un área de oportunidad o mejora; en relación con el resto de los alumnos, aportando evidencias y datos que propicien la intervención educativa dentro de una perspectiva de atención a la diversidad en el aula.

En el presente estudio interesa, no solo la estructura factorial del instrumento, sino también la equivalencia psicométrica del mismo en distintos grupos; ya que en el contexto de la comparación intergrupal, es indispensable plantearse la necesidad de llevar a cabo la adaptación de un instrumento de medida psicológica que cumpla con todos los criterios de equivalencia, pero sobre todo plantearse si la misma estructura factorial es aplicable a distintos grupos de sujetos o, de modo más genérico, a distintas poblaciones (Abalo et al., 2006)

\section{METODOLOGÍA}

\section{Participantes}

La muestra de 1181 participantes, 592 (50.1\%) alumnos de ingeniería y 589 (49.9\%) de ciencias sociales, se obtuvo mediante un muestreo por conveniencia, tratando de abarcar la representatividad de las diferentes licenciaturas de ingeniería y ciencias sociales que se ofrecen en la Universidad Autónoma de Chihuahua.

La muestra de alumnos de Ingeniería constituida por 592 participantes; 143 (24.2\%) mujeres y 449 $(75.8 \%)$ hombres. Las edades fluctúan entre los 17 y 20 años, con una media de 18.16 y una desviación estándar de 0.73 años. 
La muestra de alumnos de Ciencias Sociales compuesta por 589 participantes; 376 (63.8\%) mujeres y $213(36.2 \%)$ hombres. Las edades fluctúan entre los 17 y 20 años, con una media de 18.24 y una desviación estándar de 0.74 años.

\section{Instrumento}

Escala Autoeficacia en Conductas Académicas (EACA), encuesta tipo Likert, asistida por computadora, de 13 ítems relacionados con conductas académicas; donde el encuestado responde, en una escala de 0 a 10, la frecuencia con que actualmente, en forma ideal y si se esfuerza en cambiar, realizaría o manifestaría una acción (Blanco et al., 2011). Se eligió este tipo de encuesta por ser fácil de construir y de aplicar; además, de proporcionar una buena base para una primera ordenación de los individuos en la característica que se mide; en nuestro caso autoeficacia (Nunnally y Bernstein, 1995; Raviolo et al., 2010).

Aún cuando cada sujeto respondió a los 13 ítems del instrumento en tres escenarios distintos: Escenario actual, respondiendo en el contexto: actualmente con qué frecuencia realiza determinada conducta o se percibe con determinada característica. Escenario ideal, respondiendo en el contexto: con qué frecuencia desearía realizar determinada conducta o percibirse con determinada característica. Escenario de cambio, respondiendo en el contexto: si me esfuerzo en cambiar con qué frecuencia realizaría determinada conducta o me percibiría con determinada característica. En el análisis psicométrico solo se utilizaron las respuestas al primer escenario.

\section{Procedimiento}

Se invitó a participar en el estudio a los alumnos de primer ingreso de las licenciaturas de ingeniería y ciencias sociales que se ofrecen en la Universidad Autónoma de Chihuahua; los que aceptaron participar firmaron la carta de aceptación correspondiente. Luego se aplicó el instrumento antes descrito por medio de una computadora personal y utilizando el módulo administrador del instrumento del editor de escalas versión 2.0 (Blanco et al., 2007), en una sesión de aproximadamente 20 minutos; en los laboratorios o centros de cómputo de las unidades académicas participantes. Al inicio de cada sesión se hizo una pequeña introducción sobre la importancia de la investigación y de cómo acceder al instrumento. Las instrucciones de cómo responder, a cada uno de los 13 ítems, se encontraban en las primeras pantallas; antes del primer ítem del instrumento. Al término de la sesión se les agradeció su participación.

Una vez aplicado el instrumento se procedió a recopilar los resultados por medio del módulo generador de resultados del editor de escalas versión 2.0 (Blanco et al., 2007).

\section{Análisis de datos}

El análisis psicométrico se realizó en dos etapas: 1) análisis factorial exploratorio y 2) análisis factorial confirmatorio y de invarianza factorial; con el fin de obtener una prueba que presente las mejores propiedades para la conformación de los puntajes de autoeficacia en conductas académicas en universitarios de ingeniería y de ciencias sociales.

Para determinar el número mínimo de factores comunes capaces de reproducir, de un modo satisfactorio, las correlaciones observadas entre los ítems del instrumento, se realizaron sendos análisis factoriales exploratorios con las muestras de ingeniería y de ciencias sociales, a partir del método de factores principales, tomando como base el criterio de Kaiser-Guttman (Costello y Osborne, 2005), además de que para garantizar una adecuada representación de las variables (ítems), solo se conservaron aquéllos cuya comunalidad, o proporción de su varianza explicada por la solución factorial, fuera superior a .45; después de una rotación varimax (Costello y Osborne, 2005). Luego mediante el coeficiente a de cronbach se estimó la consistencia interna para cada factor retenido como una medida de su fiabilidad (Nunnally y Bernstein, 1995; Elosua y Zumbo, 2008). 
Para conducir el análisis factorial confirmatorio para cada muestra, se utilizó el software AMOS 16 (Arbuckle, 2007), las varianzas de los términos de error fueron especificados como parámetros libres, en cada variable latente (factor) se fijó uno de los coeficientes estructurales asociados a uno, para que su escala sea igual a la de una de las variables superficiales (ítems). El método de estimación empleado fue el de Máxima Verosimilitud; siguiendo la recomendación de Thompson (2004), en el sentido de que cuando se emplea análisis factorial confirmatorio se debe corroborar no sólo el ajuste de un modelo teórico sino que es recomendable comparar los índices de ajuste de varios modelos alternativos para seleccionar el mejor.

Para evaluar el ajuste del modelo se emplearon el estadístico Chi-cuadrado, el índice de bondad de ajuste (GFI), el residuo cuadrático medio (RMR), el error cuadrático medio de aproximación (RMSEA) y el índice de validación cruzada esperada (ECVI) como medidas absolutas de ajuste. El índice de bondad ajustado (AGFI), el Índice Tucker-Lewis (TLI), el Índice del ajuste normal (NFI) y el índice de bondad de ajuste comparativo (CFI) como medidas de ajuste incremental. El índice de ajuste normado de parsimonia (PNFI), el índice de calidad de ajuste de parsimonia (PGFI), la razón de Chi-cuadrado sobre los grados de libertad (CMIN/GL) y el Criterio de Información de Akaike (AIC) como medidas de ajuste de parsimonia (Gelabert et al., 2011; Leighton et al., 2007).

Para comprobar la invarianza factorial de la escala autoeficacia en conductas académicas entre alumnos de ingeniería y alumnos de ciencias sociales, se realizaron una serie de análisis factoriales confirmatorios multimuestra mediante el software AMOS 16 (Arbuckle, 2007).

\section{RESULTADOS}

\section{Análisis factorial exploratorio}

Para determinar el número mínimo de factores comunes capaces de reproducir, de un modo satisfactorio, las correlaciones observadas entre los 13 ítems del instrumento empleado, se realizó, un primer análisis exploratorio de Componentes Factoriales con los datos de la muestra de Ingeniería. La significación del Test de Barlett (2512.569; $p<.0001)$ y el KMO (.864) mostraron una adecuada correlación entre los ítems y una buena adecuación muestral respectivamente. Detectándose, después de una rotación varimax, una estructura de tres factores; el conjunto de los factores seleccionados explicaron el $58.96 \%$ de la varianza (Tablas 1 y 2 ).

Un segundo análisis exploratorio de Componentes Factoriales con los datos de la muestra de Ciencias Sociales; reveló de nuevo una estructura de tres factores. El conjunto de los tres factores explicaron el $61.98 \%$ de la varianza (Tablas 1 y 2). La significación del Test de Barlett (3030.467; $\mathrm{p}<$.0001) y el KMO (.898) muestran una adecuada correlación entre los ítems y una buena adecuación muestral respectivamente.

Tabla 1: Autovalores y porcentaje de varianza explicada por los factores retenidos. Análisis factorial exploratorio para alumnos de ingeniería y ciencias sociales. Soluciones rotadas.

\begin{tabular}{|l|ccc|ccc|}
\hline \multirow{2}{*}{ Factor } & \multicolumn{3}{|c|}{ Ingeniería } & \multicolumn{3}{c|}{ Ciencias Sociales } \\
\cline { 2 - 7 } & Autovalor & \% varianza & \% acumulado & Autovalor & \% varianza & \% acumulado \\
\hline 1. Atención & 2.60 & 20.04 & 20.04 & 2.97 & 22.91 & 22.91 \\
2. Excelencia & 2.52 & 19.40 & 39.44 & 2.66 & 20.50 & 43.41 \\
3. Comunicación & 2.53 & 19.52 & 58.96 & 2.41 & 18.57 & 61.98 \\
\hline
\end{tabular}

Fiabilidad de las subescalas (consistencia interna)

Las subescalas (factores) resultantes en los análisis factoriales exploratorios, de ambas muestras, poseen alfas superiores a .75 , evidenciando una consistencia interna adecuada para este tipo de subescalas, particularmente si se considera el número reducido de ítems (Tabla 3 ). 
Tabla 2: Ítems agrupados por factor. Análisis factorial exploratorio para alumnos de ingeniería y ciencias sociales. Soluciones rotadas.

\begin{tabular}{|c|c|c|c|c|c|c|}
\hline \multirow{2}{*}{ Ítem } & \multicolumn{3}{|c|}{ Ingeniería } & \multicolumn{3}{|c|}{ Ciencias Sociales } \\
\hline & 1 & 2 & 3 & 1 & 2 & 3 \\
\hline $\begin{array}{l}2 \text { Escucho con atención cuando el profesor aclara una duda } \\
\text { a un compañero }\end{array}$ & .79 & & & .76 & & \\
\hline 7 Pongo atención cuando los profesores dan la clase & .71 & & & .66 & & \\
\hline $\begin{array}{l}13 \text { Escucho con atención las preguntas y comentarios de mis } \\
\text { profesores }\end{array}$ & .69 & & & .69 & & \\
\hline 8 Pongo atención cuando un compañero expone en clase & .63 & & & .63 & & \\
\hline $\begin{array}{l}3 \text { Escucho con atención las preguntas y aportaciones de mis } \\
\text { compañeros }\end{array}$ & .59 & & & .76 & & \\
\hline 11 Entrego puntualmente los trabajos que se me encargan & & .81 & & & .83 & \\
\hline 1 Cumplo con las tareas que se me asignan & & .79 & & & .75 & \\
\hline 12 Soy cumplido en cuanto a mi asistencia & & .70 & & & .70 & \\
\hline $\begin{array}{l}10 \text { Me preparo para mis exámenes apoyándome en los } \\
\text { apuntes de clase, el texto del curso y lecturas adicionales }\end{array}$ & & .66 & & & .66 & \\
\hline 4 Expreso mis ideas con claridad & & & .82 & & & .79 \\
\hline $\begin{array}{l}9 \text { En caso de desacuerdo soy capaz de entablar un diálogo } \\
\text { con mis profesores }\end{array}$ & & & .77 & & & .76 \\
\hline 5 Hago comentarios y aportaciones pertinentes & & & .76 & & & .64 \\
\hline $\begin{array}{l}6 \text { Me siento bien con mi propio desempeño cuando hablo } \\
\text { enfrente de una clase o grupo de gente }\end{array}$ & & & .70 & & & .75 \\
\hline
\end{tabular}

Tabla 3: Coeficientes de consistencia interna de los factores obtenidos en los análisis factoriales exploratorios para alumnos de ingeniería y ciencias sociales.

\begin{tabular}{lcc}
\hline Factor & a Ingeniería & a Ciencias Sociales \\
\hline 1. Atención & .781 & .829 \\
2. Excelencia & .770 & .789 \\
3. Comunicación & .785 & .780 \\
\hline
\end{tabular}

\section{Análisis factorial confirmatorio}

Ahora, mediante el análisis factorial confirmatorio sobre los datos de las muestras de alumnos de Ingeniería y de Ciencias Sociales, se contrasta la solución factorial resultante de los análisis factoriales exploratorios realizados; con el fin de obtener modelos congenéricos y, al mismo tiempo, probar la validez de constructo de las dimensiones y variables estudiadas.

De acuerdo a los resultados de la Tabla 4 el análisis factorial confirmatorio de 13 ítems agrupados en tres factores en la muestra de Ingeniería es aceptable (GFI .953 y RMSEA .059) y de acuerdo a las medidas de ajuste incremental y de parsimonia (Tablas 5 y 6 ) significativamente superior al modelo independiente y muy similar al modelo saturado.

Por otro lado, el análisis factorial confirmatorio en la muestra de Ciencias Sociales, indica nuevamente que el modelo de medición de tres factores es aceptable (GFI .953 y RMSEA .059) y de acuerdo a las medidas de ajuste incremental y de parsimonia (ver Tablas 5 y 6) significativamente superior al modelo independiente y muy similar al modelo saturado. 
Tabla 4: Medidas absolutas de ajuste para los modelos generados. Análisis factorial confirmatorio para alumnos de ingeniería y ciencias sociales; * $p<.05$.

\begin{tabular}{lccccc}
\hline & \multicolumn{5}{c}{ Índice de Ajuste } \\
\cline { 2 - 6 } Modelo & $\chi^{2}$ & GFI & RMR & RMSEA & ECVI \\
\hline Independiente (13 ítems) & $3057.331^{*}$ & .365 & 0.977 & .255 & 5.244 \\
Saturado (13 ítems) & 0 & 1 & 0 & & 0.310 \\
3 factores 13 ítems & $195.486^{*}$ & .952 & 0.114 & .061 & 0.431 \\
\hline & Solución factorial para Ciencias Sociales & & \\
Independiente (13 ítems) & $2534.728^{*}$ & .447 & 0.849 & .223 & 4.333 \\
Saturado (13 ítems) & 0 & 1 & 0 & & 0.308 \\
3 factores 13 ítems & $191.022^{*}$ & .953 & 0.115 & .059 & 0.421 \\
\hline
\end{tabular}

Tabla 5: Medidas de ajuste incremental para los modelos generados. Análisis factorial confirmatorio para alumnos de ingeniería y ciencias sociales.

\begin{tabular}{|c|c|c|c|c|}
\hline \multirow[b]{2}{*}{ Modelo } & \multicolumn{4}{|c|}{ Índice de Ajuste } \\
\hline & AGFI & TLI & $\mathrm{NFI}$ & CFI \\
\hline \multicolumn{5}{|c|}{ Solución factorial para Ingeniería } \\
\hline Independiente (13 ítems) & .354 & 0 & 0 & 0 \\
\hline Saturado (13 ítems) & & & 1 & 1 \\
\hline 3 factores 13 ítems & .931 & .934 & .925 & .947 \\
\hline \multicolumn{5}{|c|}{ Solución factorial para Ciencias Sociales } \\
\hline Independiente (13 ítems) & .260 & 0 & 0 & 0 \\
\hline Saturado (13 ítems) & & & 1 & 1 \\
\hline 3 factores 13 items & .930 & .944 & .936 & .955 \\
\hline
\end{tabular}

Tabla 6: Medidas de ajuste de parsimonia para los modelos generados. Análisis factorial confirmatorio para alumnos de ingeniería y ciencias sociales.

\begin{tabular}{lcccc}
\hline & \multicolumn{4}{c}{ Índice de Ajuste } \\
\cline { 2 - 4 } Modelo & PNFI & PGFI & CMIN/GL & AIC \\
\hline & Solución factorial para Ingeniería \\
Independiente (13 ítems) & 0 & .383 & 32.497 & 2560.728 \\
Saturado (13 ítems) & 0 & & 3.081 & 182.000 \\
3 factores 13 ítems & .735 & .649 & 249.022 \\
\hline & Solución factorial para Ciencias Sociales & \\
Independiente (13 ítems) & 0 & .313 & 39.197 & 3083.331 \\
Saturado (13 ítems) & 0 & & 3.153 & 253.486 \\
3 factores 13 ítems & .744 & .649 &
\end{tabular}


En la figura 1 se presenta el modelo de medición del primer análisis factorial confirmatorio (alumnos de ingeniería) para los 13 ítems agrupados en tres factores, incluyendo los coeficientes de regresión estandarizados entre ítems y factores y las saturaciones factoriales estandarizadas (comunalidades) de cada uno de los ítems.

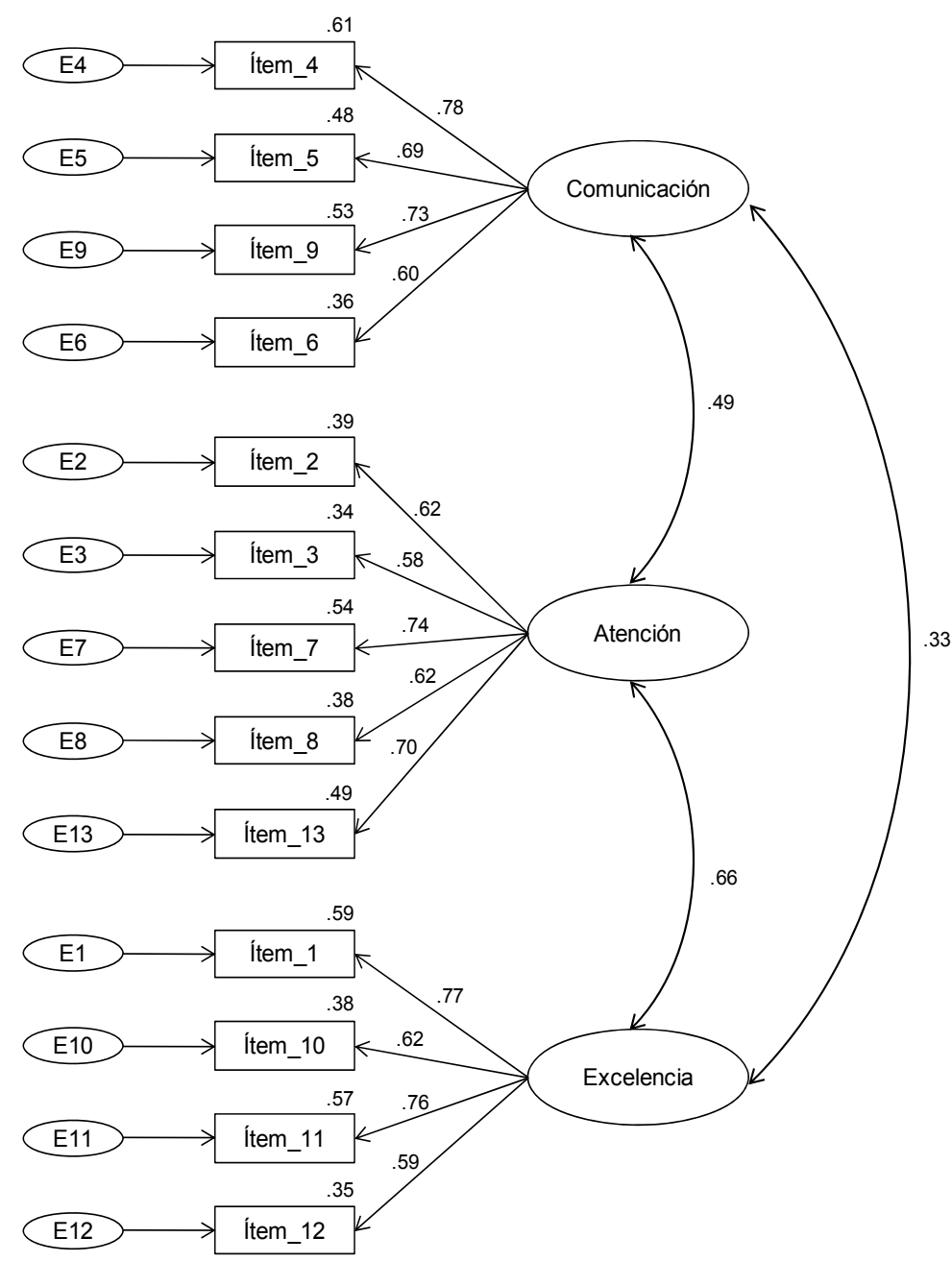

Fig. 1: Modelo de medición para la escala. Análisis factorial confirmatorio alumnos de Ingeniería. Fuente: de los autores.

El factor Comunicación presenta una saturación factorial mínima de .36, correspondiente al ítem 6 mientras que el resto de ítems explicados por el factor presentan saturaciones por encima de .45. En el factor Atención, todos los ítems resultan bien explicados a partir del constructo hipotetizado, con una saturación mínima de .34 (ítem 3), y el resto, por encima de .35. Finalmente, el factor Excelencia, presenta en general, saturaciones factoriales estandarizadas elevadas (mayores a .55), salvo en el caso de los ítems 10 y 12 , con saturaciones moderadas (.38 y .35 respectivamente).

Por su parte, todos los factores correlacionan entre sí de forma estadísticamente significativa $(p<.01)$. Las correlaciones entre Comunicación y los factores Atención y Excelencia pueden considerarse moderadas, con valores de .49 y .33 respectivamente, mientras que la relación entre estos dos últimos factores es alta (.66); lo cual muestra que a medida que aumenta la autoeficacia percibida en alguno de los factores, también aumenta en los otros dos.

En la figura 2 se presenta el modelo de medición del segundo análisis factorial confirmatorio (alumnos de ciencias sociales) para los 13 ítems agrupados en tres factores, incluyendo los coeficientes de regresión estandarizados entre ítems y factores y las saturaciones factoriales estandarizadas (comunalidades) de cada uno de los ítems. 


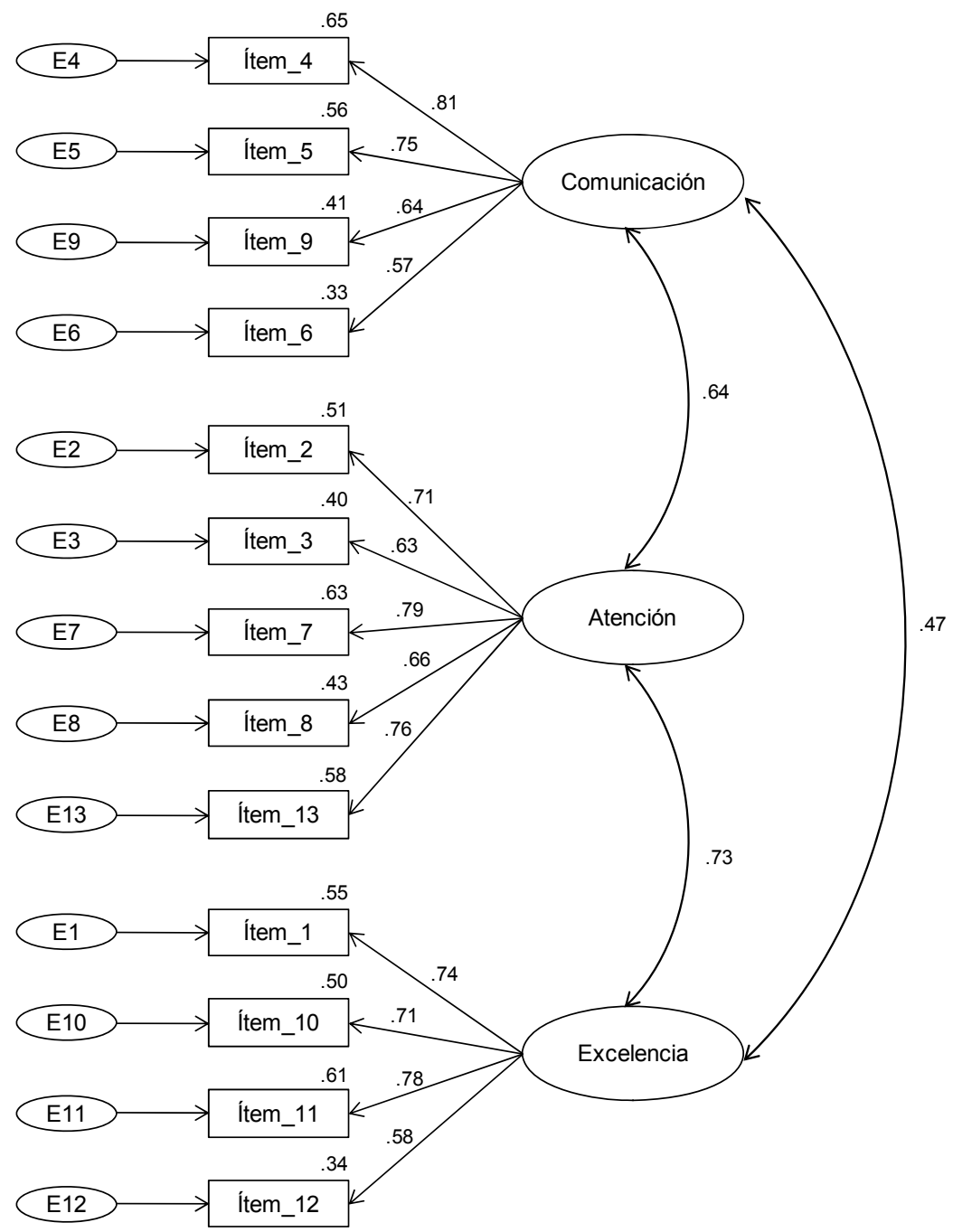

Fig. 2: Modelo de medición para la escala. Análisis factorial confirmatorio alumnos de Ciencias Sociales. Fuente: de los autores.

El factor Comunicación, presenta en general, saturaciones factoriales estandarizadas elevadas (mayores de .40), salvo en el caso del ítem 6, con una saturación moderada (.33). En el factor Atención, todos los ítems resultan bien explicados a partir del constructo hipotetizado, con saturaciones entre .40 y .63. Finalmente, el factor Excelencia, presenta una saturación factorial moderada de .34, correspondiente al ítem 12 mientras que el resto de ítems explicados por el factor presentan saturaciones de .50 o más.

Por su parte, todos los factores correlacionan entre sí de forma estadísticamente significativa $(p<.01)$. Las correlaciones entre Comunicación y los factores Atención y Excelencia pueden considerarse de altas a moderadas, con valores de .64 y .47 respectivamente, mientras que la relación entre estos dos últimos factores es alta (.73), lo cual muestra que a medida que aumenta la autoeficacia percibida en alguno de los factores, también aumenta en los otros dos.

Invarianza de la estructura factorial entre alumnos de ingeniería y ciencias sociales

Para analizar la invarianza factorial del cuestionario se siguieron las recomendaciones de Abalo et al. (2006) estimando el mismo modelo en ambas muestras; los índices de ajuste obtenidos (Tabla 7) permiten aceptar la equivalencia de los modelos de medida básicos entre las dos muestras. Aunque el valor de Chi-cuadrado excede al exigido para aceptar la hipótesis de invarianza, el resto de índices contradicen esta conclusión (GFI .953; CFI .952; RMSEA .042; AIC 502.508) lo que nos permite aceptar el modelo base de la invarianza (modelo sin restricciones). 
Añadiendo al modelo base restricciones sobre las cargas factoriales caracterizamos la invarianza métrica. Los valores que se recogen en la tabla 7 permiten aceptar este nivel de invarianza. El índice de ajuste general (GFI= .951) y el error cuadrático medio de aproximación (RMSEA $=.041)$ siguen aportando información convergente en esta dirección. Además, el criterio de información de Akaike $(A I C=496.448)$ y el índice comparativo de Bentler $(C F I=.951)$ no sufren grandes variaciones respecto al modelo anterior. Haciendo uso del criterio para la evaluación de los modelos anidados propuesto por Cheung y Rensvold (2002), quiénes sugieren que si el cálculo de la diferencia de los CFI de ambos modelos anidados disminuye en .01 o menos, se da por bueno el modelo restringido y por tanto el cumplimiento de la invarianza factorial; la diferencia entre CFIs obtenida .001 permite aceptar el modelo de invarianza métrica. Podemos concluir hasta ahora que las cargas factoriales son equivalentes en las dos muestras.

Una vez demostrada la invarianza métrica entre las muestras, pasamos a evaluar la equivalencia entre interceptos (invarianza factorial fuerte). Los índices (Tabla 7) muestran un buen ajuste de este modelo, tanto evaluado de modo independiente como analizándolo respecto a su anidamiento con el modelo de invarianza métrica. La diferencia entre los índices comparativos de Bentler es de .003; el índice de ajuste general es .948 y el error cuadrático medio de aproximación es .041. Aceptada la invarianza fuerte, los dos modelos evaluados son equivalentes respecto a los coeficientes factoriales y a los interceptos.

Tabla 7: Índices de bondad de ajuste de cada uno de los modelos puestos a prueba en la invarianza factorial; * $p<.05$.

\begin{tabular}{lccccccc}
\hline \multicolumn{1}{c}{ Modelo } & \multicolumn{2}{l}{$\begin{array}{l}\text { Índice de } \\
\text { Ajuste }\end{array}$} & & & & & \\
& \multicolumn{1}{c}{$\chi^{2}$} & gl & GFI & NFI & CFI & RMSEA & AIC \\
\hline Modelo sin restricciones & $386.508^{*}$ & 124 & .953 & .931 & .952 & .042 & 502.508 \\
Invarianza métrica & $400.448^{*}$ & 134 & .951 & .928 & .951 & .041 & 496.448 \\
Invarianza factorial fuerte & $421.658^{*}$ & 140 & .948 & .925 & .948 & .041 & 505.658 \\
\hline
\end{tabular}

Contrastes de las medias de los factores entre alumnos de ingeniería y ciencias sociales

Una vez comprobada la invarianza factorial, las diferencias entre las medias de los factores de los dos grupos se estimaron tomando como referente la muestra de ingeniería, fijando en 0 el valor de las medias para dicha muestra y estimando libremente el valor de las medias para la muestra de ciencias sociales. Las restricciones sobre los coeficientes de regresión e interceptos, requeridos para los contrastes entre las medias se realizaron automáticamente mediante el software AMOS 16 (Arbuckle, 2007). Los resultados de las comparaciones entre medias indicaron que la media de los factores Comunicación y Excelencia fue significativamente mayor $(0.282, p<0.01$ y $0.278, p$ $<0.01$ respectivamente), en los alumnos de ciencias sociales; y sin diferencia en el Factor Atención.

\section{CONCLUSIONES}

De los resultados mostrados, de su análisis y de su discusión, y tomando en cuenta que el objetivo principal de este estudio fue el de examinar la estructura factorial y la medición de la invariancia de dicha estructura en alumnos de ingeniería y ciencias sociales, se pueden obtener las siguientes conclusiones:

1) El Análisis factorial exploratorio del instrumento autoeficacia en conductas académicas llevado a cabo en cada una de las muestras estudiadas puso de manifiesto una estructura de tres factores: Atención, Comunicación y Excelencia.

2) Los factores en ambas muestras evidenciaron una consistencia interna adecuada, particularmente si se considera el número reducido de ítems en cada uno de ellos. 
3) El Análisis Factorial Confirmatorio indicó que el ajuste de los datos al modelo teórico de 13 ítems agrupados en tres factores es aceptable. Al mismo tiempo que los tres factores así obtenidos presentan en general saturaciones factoriales estandarizadas adecuadas. Por su parte los tres factores correlacionan entre sí de forma positiva y estadísticamente significativa lo cual muestra que a medida que aumenta la autoeficacia percibida en alguno de los factores, también aumenta en los otros dos.

4) Conjuntamente con todo lo antes dicho, los resultados del análisis de la invarianza factorial entre las muestras; indican una alta congruencia entre pares de factores. Lo que sugiere la existencia de fuertes evidencias de la validación cruzada de la medida y por tanto de la estabilidad de la estructura, hasta que no se demuestre lo contrario.

5) Las comparaciones entre los grupos reflejaron diferencias significativas, a favor de los alumnos de ciencias sociales, en las medias de los factores Comunicación y Excelencia. Lo que parece indicar que los alumnos de ciencias sociales se perciben un poco más autoeficaces que los alumnos de ingeniería en relación a dichos factores.

En síntesis, el análisis de las propiedades psicométricas, ha mostrado que una estructura trifactorial es viable y apropiada de acuerdo a los requisitos psicométricos establecidos cuando los informantes son los propios alumnos. La estructura de tres factores, atendiendo a criterios estadísticos y sustantivos, ha mostrado adecuados indicadores de ajuste, de fiabilidad y de validez. Sin embargo, consideramos que más estudios son necesarios con el fin de corroborar o refutar los datos obtenidos en la presente investigación.

\section{REFERENCIAS}

Abalo, J., Lévy, J., Rial, A., y Varela, J., Invarianza factorial con muestras múltiples. En J. Lévy (Ed.), Modelización con Estructuras de Covarianzas en Ciencias Sociales pp. 259-278. Netbiblo, Madrid (2006).

Arbuckle, J., AMOS users guide version 16.0 (2007).

Bandura, A., Self-efficacy mechanism inhuman agency, American Psychologist, 37(2), 122-147 (1982).

Bandura, A., Self-efficacy: The exercise of Control, Freeman, New York (1997).

Blanco, H., Martínez, M., Zueck, M. A. y Gastélum, G., Análisis psicométrico de la escala autoeficacia en conductas académicas en universitarios de primer ingreso, Actualidades Investigativas en Educación, 11(3), 1-27 (2011).

Blanco, H. y otros siete autores, Editor para la construcción y aplicación de escalas por medio de una PC, Tecnociencia Chihuahua, 1(1), 55-59 (2007).

Carbonero, M. A. y Merino, E., Autoeficacia y madurez vocacional, Psicothema, ISSN: 1886-144X (en línea), 16(2), 229-234, 2008. http://www.psicothema.com/pdf/1187.pdf. Acceso: 3 de agosto (2011).

Cheung, G. W. y Rensvold, R. B., Evaluating goodness-of-fit indexes for testing measurement invariance, Structural Equation Modeling, 9, 233-255 (2002).

Castañeda, S., Pineda, M. L., Gutierrez, E., Romero, N. y Peñalosa, E., Construcción de instrumentos de estrategias de estudio, autorregulación y epistemología personal. Validación de constructo, Revista Mexicana de Psicología, 27(1), 77-85 (2010). 
Costello, A. y Osborne, J., Best practices in exploratory factor analysis: four recommendations for getting the most from your analysis. Practical Assessment, Research and Evaluation, ISSN: 15317714, 10(7), 1-9, 2005. http://pareonline.net/pdf/v10n7.pdf. Acceso: 9 de Mayo (2011).

Elosua, P. y Zumbo, B., Coeficientes de fiabilidad para escalas de respuesta categórica ordenadas, Psicothema, ISSN: 1886-144X (en línea), 20(4), 896-901, 2008. http://www.psicothema.com/pdf/3572.pdf. Acceso: 3 de agosto (2011).

Gelabert, E., García-Esteve, L., Martín-Santos, R., Gutiérrez, F., Torres, A. y Subirà, S., Psychometric properties of the Spanish version of the Frost Multidimensional Perfectionism Scale in women, Psicothema, ISSN: 1886-144X (en línea), 23(1), 133-139, 2011. http://www.psicothema.com/pdf/3861.pdf. Acceso: 3 de agosto (2011).

Leighton, J., Gokiert, R. y Cui, Y., Using Exploratory and Confirmatory Methods to Identify the Cognitive Dimensions in a Large-Scale Science Assessment, International Journal of Testing, 7(2), 141-189 (2007).

León-Rubio, J. M., Cantero, F. J. y León-Pérez, J. M., Diferencias del rol desempeñado por la autoeficacia en el burnout percibido por el personal universitario en función de las condiciones de trabajo, Anales de Psicología, 27(2), 518-526. (2011).

Nunnally, J. y Bernstein, I., Teoría Psicométrica, McGraw-Hill, México (1995).

Ornelas, M., Blanco, H., Gastélum, G. y Chávez, A., Autoeficacia percibida en conductas académicas en mujeres universitarias, Form. Univ., 5(2), en prensa (2012).

Ornelas, M., Blanco, H., Rodríguez, J. M. y Flores, F. J., Análisis Psicométrico de la Escala Autoeficacia en Conductas de Cuidado de la Salud Física en Universitarios de Primer Ingreso, Form. Univ., 4(6), en prensa (2011).

Pérez, E., Lescano, C., Zalazar, P., Furlám, L. y Martínez, M., Desarrollo y análisis psicométricos de un Inventario de Autoeficacia para Inteligencias Múltiples en Niños Argentinos. Psicoperspectivas, ISSN: 0718-6924 (en línea), 10 (1), 169-189, 2011. http://www.psicoperspectivas.cl/index.php/psicoperspectivas/article/viewFile/141/129. Acceso: 3 de agosto (2011).

Raviolo, A., Ramirez, P., López, E. A. y Aguilar, A., Concepciones sobre el Conocimiento y los Modelos Científicos: Un Estudio Preliminar, Form. Univ., ISSN: 0718-5006 (en línea), 3(5), 29-36, 2010. http://www.scielo.cl/pdf/formuniv/v3n5/art05.pdf. Acceso: 3 de agosto (2011).

Sansinenea, E. y otros seis autores, Autoconcordancia y autoeficacia en los objetivos personales: ¿Cuál es su aportación al bienestar?, Anales de Psicología, 24(1), 121-128 (2008).

Thompson, B., Exploratory and Confirmatory Factor Analysis. Understanding concepts and applications, American Psychological Association, Washington, D C: (2004).

Vera, M., Salanova, M. y Martín-del-Río, B., Self-efficacy among university faculty: how to develop an adjusted scale, Anales de Psicología, 27(3), 800-807 (2011).

Wolters, C., Advancing achievement goal theory: using goal structures and goal orientations to predict students motivation, cognition and achievement, Journal of Educational Psychology, 96(2), 236-250 (2004).

Zimmerman, B. y Kitsantas, A., Homework practice and academic achievement. The mediating role of self-efficacy and perceived responsibility beliefs, Contemporany Educational Psychology, 30(4), 397-417 (2005). 
